\title{
Numerical simulation of goaf gas distribution law in different ventilation modes
}

\author{
LI Sheng Zhou ${ }^{1,2, a}$ LIU Jun ${ }^{1,2, b}$ \\ ${ }^{1}$ State Key Laboratory of Gas Disaster Detecting, Preventing and Emergency Controlling \\ ${ }^{2}$ Chongqing branch of Chinese coal research institute Co., Ltd \\ alszaxx|@qq.com, b274705046@qq.com
}

Keywords: the goaf; gas distribution law; numerical model

Abstract. By analyzing the overlying strata caving characteristics and properties of porous media that a heterogeneous porous media properties mined crack rectangle landings is formed in the mined-out area. Introduction of translation-diffusion equation and to simulate the flow of gas diffusion behavior; by the application of Navier-Stokes equations and Brinkman equations building face and goaf gas flow model to establish a physical model of gas flow in coal mining face. COMSOL Multiphysics numerical software for the study of the mined-out area under different ventilation mode gas distribution numerical simulation results show that: the tail roadway and high drainage roadway can improve the upper corner gas overrun problem.

\section{Introduction}

Mine gas concentration exceeding limits accident occurred mainly in the coalface, especially on the upper corner. Coalface gas mainly has three sources: the coal seam gas, gas of adjacent seam and gob gas. Gob gas is the main source of emission in these three sources, or even account for more than $75 \%$ of the entire gas sources, so gob gas emission has been a major risk ${ }^{[1-2]}$ in mine production. Therefore, the analysis of gob gas distribution has important significance for gas control in mining face ${ }^{[3-5]}$.

The domestic and foreign scholars have done a lot of research for goaf gas seepage from the mechanism of gas flow in coal seams,established mathematical model of gas flow in the gas medium parameters change with time and space, namely the gas flow control equation. With the development of computer technology, using CFD (Computational Fluid Dynamics) model of goaf gas distribution research become feasible and effective. However, the gas flow in coal face is a complex process, described the basic CFD model is difficult to accurately on the coal face.

In this paper, through the analysis of overburden rock caving characteristics and properties of the porous medium, a mining fissure rectangular terrace with heterogeneous porous media properties is formed in goaf which can meet the needs of engineering precision. According to the fluid motion equation in working face and goaf and gas flow diffusion model,using Multiphysics analysis software COMSOL multiphysics, goaf gas flow and occurrence regularity of the mining process is got by the numerical simulation calculation.

\section{Equations of gas flow in porous media}

After the gob roof falling, with the mining work goes on, the overlying strata began to move and destroy, which can be divided into three areas in the transverse direction, coal wall support area and separation area, recompaction zone, respectively from bottom to top with caving zone, fracture zone and bending zone in the longitudinal direction; it can be considered that a mining fissure rectangular terrace with heterogeneous porous media properties is formed in whole rock strata which can meet the needs of engineering precision as shown in figure 1. After mining, there are a lot of voids in the mining fissure zone, voids system is composed of coal and rock mass bedding, joints and cracks. According to the definition of fluid mechanics in porous medium and its main physical and mechanical properties, the goaf is approximate to the heterogeneous porous media, therefore, the gas 
flow in the goaf can be approximately regarded as the gas flow in the porous medium, which provides a theoretical basis for the study of the gas migration law.

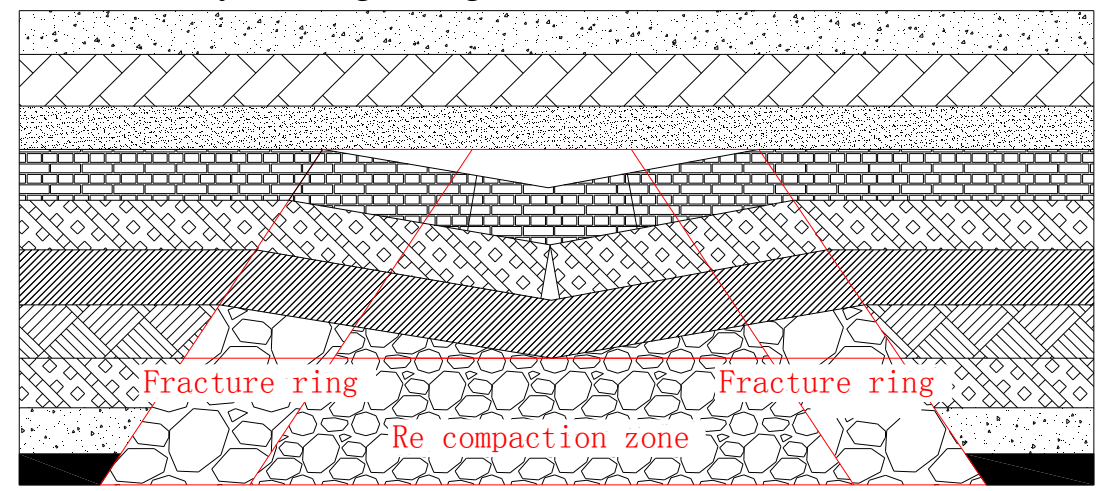

Fig. 1 Schematic diagram of the cross section of the broken ladder of the overburden rock

According to the physical and mechanical properties in each area of the mined out area, in order to facilitate research, the goaf roof fall area shall be deemed isotropic porous media. Previous researchers have done a lot of research on the permeability coefficient and porosity of the goaf and the breaking expansion coefficient of the rock. It is concluded that there is a certain relationship between the three, the Blake-Kozeny equation is ${ }^{[6]}$ :

$$
\begin{aligned}
& k=\frac{\varepsilon^{3} d_{m}^{2}}{150(1-\varepsilon)^{2}} \\
& \varepsilon=1-1 / \mathrm{K}_{\mathrm{p}}
\end{aligned}
$$

Where, $\mathrm{k}$ is the permeability coefficient; $\varepsilon$ is the porosity; $\mathrm{K}_{\mathrm{p}}$ is broken rock caving expansion coefficient, in this paper, based on the existing research results; $d_{m}$ is the mean particle diameter of the porous medium, $\mathrm{m}$.

Flow Equation. Conservation of momentum is a universal law which can be described as: in a given fluid system, the time rate of change of its momentum is equal to the sum of the forces on its role. The Navier-Stoke equation of motion is dominated by the air flow kinetic energy, and the permeability resistance of air flow in porous media is neglected, which is suitable for describing the flow pattern of the fluid in the pipeline, the coal mine roadway and the space of the working face ventilation process can be regarded as the pipe fluid flow, Navier- Stokes equation dependent variable velocity vector, respectively $\left(\mathrm{u}_{\mathrm{ns}}\right)$ and pressure $\left(\mathrm{p}_{\mathrm{ns}}\right)$, which is expressed as:

$$
\begin{aligned}
& \rho \frac{\partial u_{n s}}{\partial t}+\left(\rho u_{n s} \cdot \nabla\right) u_{n s}=-\nabla p_{n s}+\nabla\left(\eta\left(\nabla u_{n s}+\left(\nabla u_{n s}\right)^{T}\right)\right)+F \\
& \nabla \cdot u_{n s}=0
\end{aligned}
$$

Wherein, $\eta$ is coefficient of dynamic viscosity, Pa.s; $u_{n s}$ as the velocity vector, $\mathrm{m} / \mathrm{s} ; \rho$ is the fluid density, $\mathrm{kg} / \mathrm{m}^{3}$; $\mathrm{p}_{\mathrm{ns}}$ is pressure, $\mathrm{Pa} ; \mathrm{F}$ fluid resistance; $\nabla$ is Laplas operator.

Mining goaf formed by a large number of smoke of gangue and voids, which can be regarded as porous media. The flow of gas in the mined out area can be described by the Brinkman ${ }^{[7-9]}$ equation which is suitable for describing the transition zone between the Darcy flow and the fluid flow in the porous media. Brinkman equations describe fluid in porous media, due to fluid motion rule under fluid velocity kinetic energy, fluid pressure and gravity, the gob caving zone romantic to take into account the characteristics of fluid pressure gradient and motion, more suitable for goaf falling movement of airflow field. The dependent variables of the Brinkman equation are velocity vector $\left(\mathrm{u}_{\mathrm{br}}\right)$ and pressure $\left(\mathrm{p}_{\mathrm{br}}\right)$.

$$
\frac{\rho}{\varepsilon}\left(\frac{\partial u_{b r}}{\partial t}\right)+\left(\frac{\eta}{k}\right) u_{b r}=-\nabla p_{b r}+\nabla\left(\frac{\eta}{\varepsilon}\left(\nabla u_{b r}+\left(\nabla u_{b r}\right)^{T}\right)\right)+F
$$




$$
\nabla \cdot u_{b r}=0
$$

Wherein, $\mathrm{u}_{\mathrm{br}}$ the velocity vector, $\mathrm{m} / \mathrm{s} ; \mathrm{p}_{\mathrm{br}}$ as pressure, $\mathrm{Pa} ; \varepsilon$ is the porosity; $\mathrm{k}$ is permeability.

Gas Diffusion Transport Model. Mined out area gas convective diffusion migration process, including the physical process of gas concentration diffusion and convection ventilation of two interacting. Among them, the diffusion and migration of gas in porous media can be described by the diffusion equation of porous medium::

$$
\theta_{s} \frac{\partial c}{\partial t}+\nabla \cdot\left(\theta_{s} D_{L} \nabla c+u c\right)=S_{c}
$$

Where, $\theta$ s fluid volume ratio; $\mathrm{c}$ is the concentration of dissolved, $\mathrm{kg} / \mathrm{m}^{3} ; \mathrm{D}_{\mathrm{L}}$ diffusion tensor, $\mathrm{m}^{2} / \mathrm{d}$; $\mathrm{S}_{\mathrm{c}}$ is the increase in the amount of solute per unit of time per unit volume of porous medium, that is, the relative emission rate of gas.

\section{Engineering Background And Numerical Model}

Project Background. Simulation of $4 \mathrm{~m}$ coal seam thickness, seam. In accordance with the collapse of the rectangular ladder platform divides the region characteristics into fifteen regions, the air intake and return air lane, tail lane, Gao Chou lane and working face space as a cuboid, the intake and return air lane dimensions: length $20 \mathrm{~m}$, width $3 \mathrm{M}$, high $4 \mathrm{~m}$; size: length $120 \mathrm{~m}$, width $3 \mathrm{M}$, high $4 \mathrm{~m}$. Fracture zone height gap and seam is $20 \mathrm{~m}$, rectangular ladder body length $180 \mathrm{~m}$, width $120 \mathrm{~m}$, high $60 \mathrm{~m}$, simulation geometry as shown in figure 2. Simulation of coal mining in the U type, U+L type, U type and high gas concentration distribution to Xiang three ventilation conditions.

According to the research results and previous experience, the fifteen regional body rectangular ladder permeability, expansion coefficient and porosity values according to table 1 .

Due to the distribution of goaf gas emission between various sources is not clear, so will the goaf gas emission as the uniform distribution, to various goaf gas emission sources from the average as the gas source unit volume. The quality of the gas source model is set to two, will have space for coal mining goaf of coal and coal seam gas emission sources, the other will work space for the coal wall gas source. According to information calculated seam coal face wall of gas emission rate of $7.46 \times 10^{-5}$ $\mathrm{kg} /\left(\mathrm{m}^{3} \cdot \mathrm{s}\right)$, Gobs and adjacent coal seam gas Emission total $8.72 \times 10^{-6} \mathrm{~kg} /\left(\mathrm{m}^{3} \cdot \mathrm{s}\right)$, gas density of 0.716 $\mathrm{kg} / \mathrm{m}^{3}$, measured air dynamic viscosity coefficient of $5 \times 10^{-5} \mathrm{~Pa} \cdot \mathrm{s}$.

The intake position of the inlet boundary, the inlet boundary contains airflow velocity, gas concentration, intake airflow velocity is $2 \mathrm{~m} / \mathrm{s}$, the gas concentration is 0 , that does not contain gas into the fresh air. Return air lane, tail roadway and high drainage roadway set to set pressure outlet boundary, outflow type. The rest of the solid boundary is set to the wall. According to the numerical model and parameter set, numerical simulation, model to calculate the residual convergence until now, to goaf flow field, gas distribution characteristics.

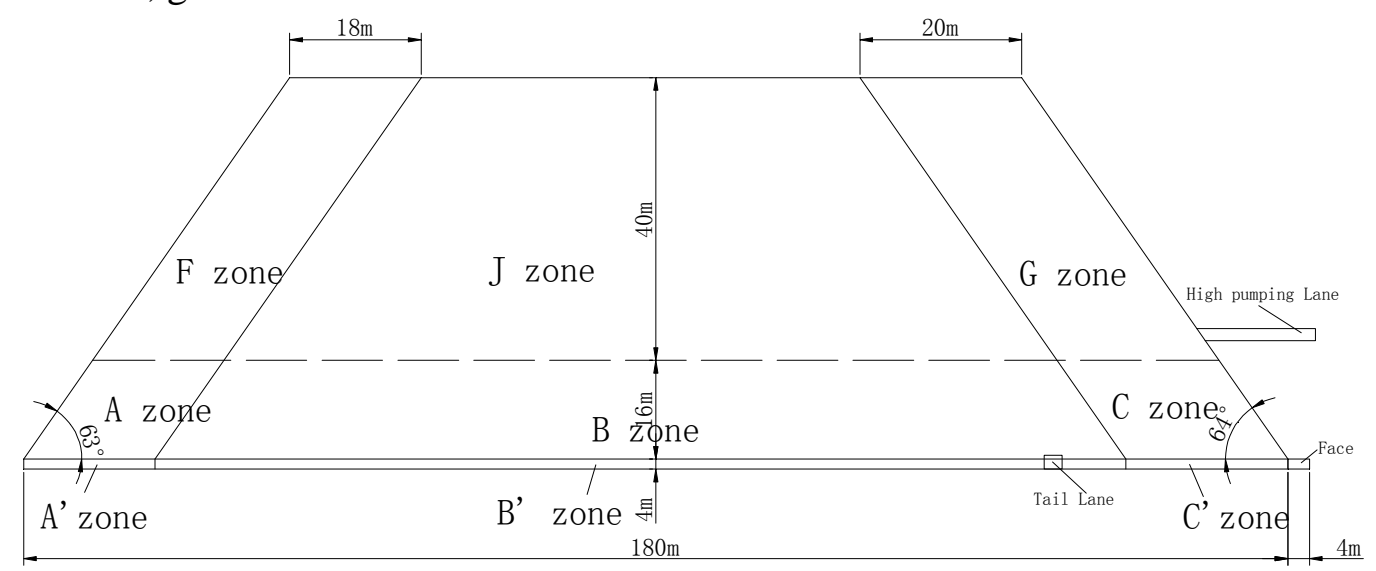

(a) model to the section size of the schematic 


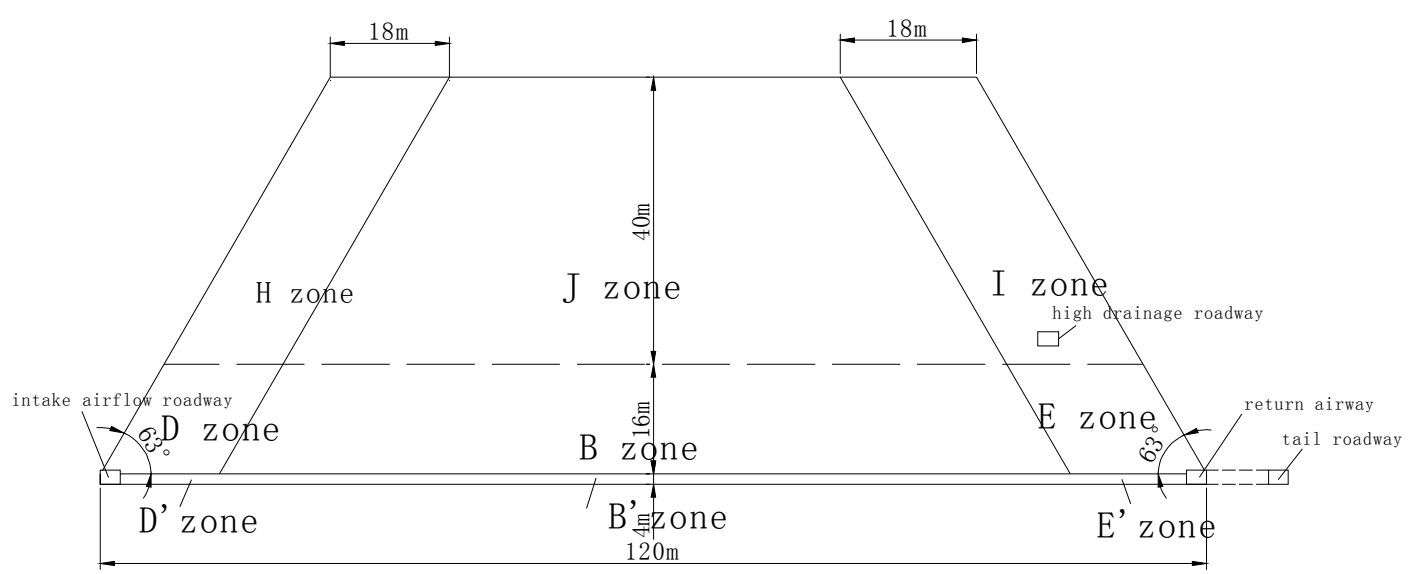

(b) model of the tendency of cross-section size sketch map

Fig. 2 Schematic diagram of model geometry

Table 1 Permeability and porosity coefficients of different regions

\begin{tabular}{|c|c|c|c|c|c|c|c|c|c|c|}
\hline \multirow{2}{*}{ Region } & A & B & $\mathrm{C}$ & $\mathrm{D}$ & $\mathrm{E}$ & \multirow{2}{*}{$\mathrm{F}$} & \multirow{2}{*}{ G } & \multirow{2}{*}{$\mathrm{H}$} & \multirow{2}{*}{ I } & \multirow{2}{*}{$\mathrm{J}$} \\
\hline & $A^{\prime}$ & $\mathrm{B}^{\prime}$ & $C^{\prime}$ & $D^{\prime}$ & $E^{\prime}$ & & & & & \\
\hline $\begin{array}{c}\text { Expansion } \\
\text { coefficient } K_{p}\end{array}$ & 1.12 & 1.05 & 1.2 & 1.12 & 1.12 & 1.08 & 1.08 & 1.08 & 1.13 & 1.07 \\
\hline The porosity $\varepsilon$ & 0.11 & 0.05 & 0.17 & 0.11 & 0.11 & 0.07 & 0.07 & 0.07 & 0.12 & 0.07 \\
\hline Permeability k/*e & 1.2 & 0.03 & 1.45 & 1.2 & 1.2 & 0.14 & 0.14 & 0.14 & 0.3 & 0.007 \\
\hline
\end{tabular}

Model And the Results Of Numerical Analysis Solver. COMSOL Multiphysics is based on finite element method, by solving the partial differential equations and partial differential equations to realize the real physical phenomena simulation. In COMSOL through the custom PDE mode can be very flexible to achieve the coupling between complex problems. In the coal seam working face ventilation process, the fresh airflow roadway follow Navier Stokes flow, part of working face air leakage into the mined out area gradient for the Brinkman flow, under appropriate assumptions software can be calculated into the goaf flow and outflow mining goaf pressure. The flow field in the working face and goaf forms a new equilibrium, which is a multi physical field coupling process of fluid flow and convection diffusion. Using the COMSOL model for solving simultaneous nonlinear steady-state model of air flow and gas diffusion, the results of gas concentration distribution in working face and goaf under three different ventilation modes are simulated, the results are shown in figure 3 .
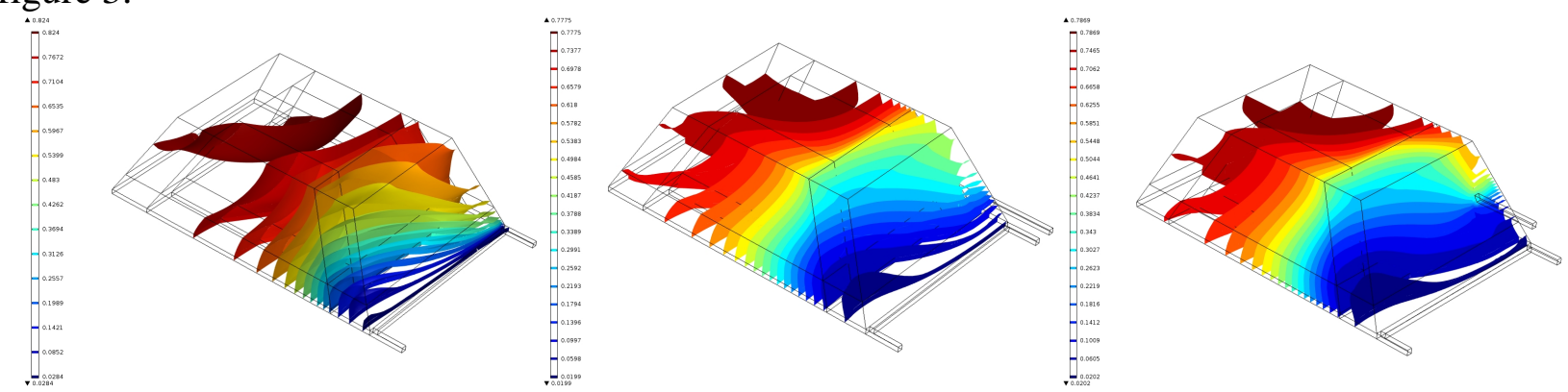

(a) $U$ type gas concentration contour plot (b) $U+L$ goaf gas concentration contour plot (c) $U$ type + high drainage roadway gas equivalent surface map

Fig. 3 gas concentration distribution in Goaf

The blue area is the low gas concentration area and the red is the high gas concentration area. By comparing the gas concentration distribution under three ventilation modes in the goaf, the following conclusions can be obtained

(1) From Figure 3 we can see that three different ventilation modes, goaf gas concentration distribution rules roughly similar. At the work surface, the gas emission by fresh air flow away, the whole gas concentration is low. Specifically, the intake side of the gas of low concentration, gas concentration is relatively high on the side of the wind lane; Influence of air leakage in the working 
face became smaller with distance from the working face and goaf methane concentration with distance to the working face of increased from.

(2) From Figure 3 (b) we can see that the effect is very obvious in the tail of the roadway. In the air flow pressure difference under the goaf of the part of the gas is diverted to a dedicated gas lane, reducing the gas concentration of the working surface, relative to the $U$ type ventilation, the maximum gas concentration from $82.4 \%$ to $78.7 \%$. It can be seen from the chart that the volume of the low gas concentration area is obviously increased, and the ventilation of the working face has a great influence on the low gas concentration area and extends to the deep part of the goaf.

(3) From Figure 3 (c) we can see that, the layout of the high level gas drainage tunnel has obvious influence on gas control, the guiding role of the air guide mining deep goaf of high concentration gas and working face of fresh air to the high drainage roadway flow, which changed the distribution of the gas concentration, and compared with $U$ type ventilation, the maximum gas concentration in the mined out area decreased from $82.4 \%$ to $77.25 \%$.

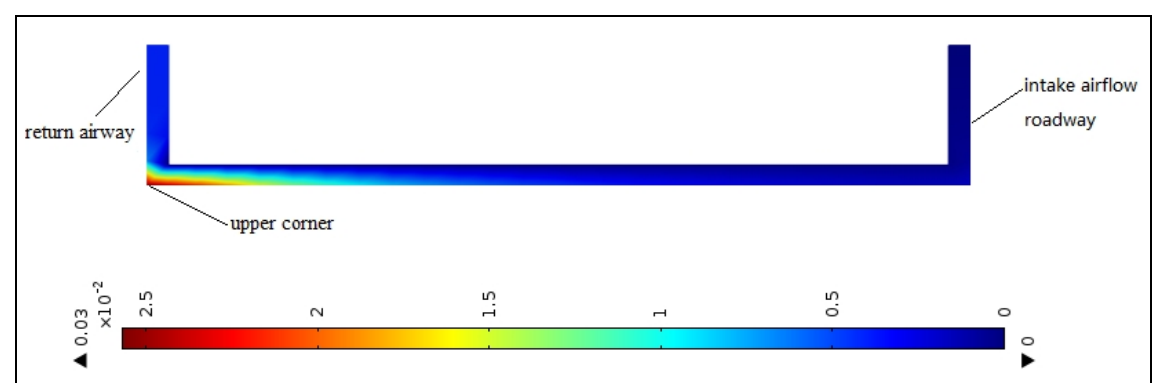

(a) Distribution of concentration of the gas in the working face of $U$ type ventilation

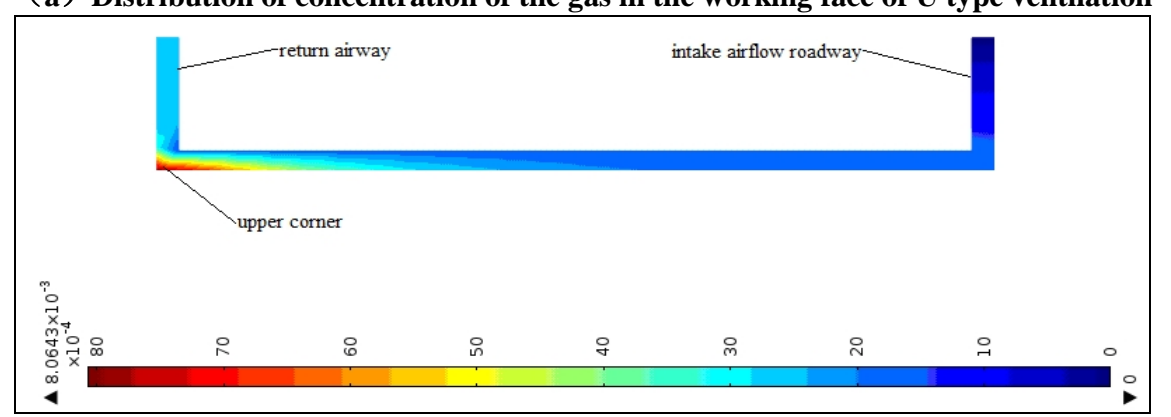

(b) Distribution of concentration of the gas in the working face of U+Ltype ventilation

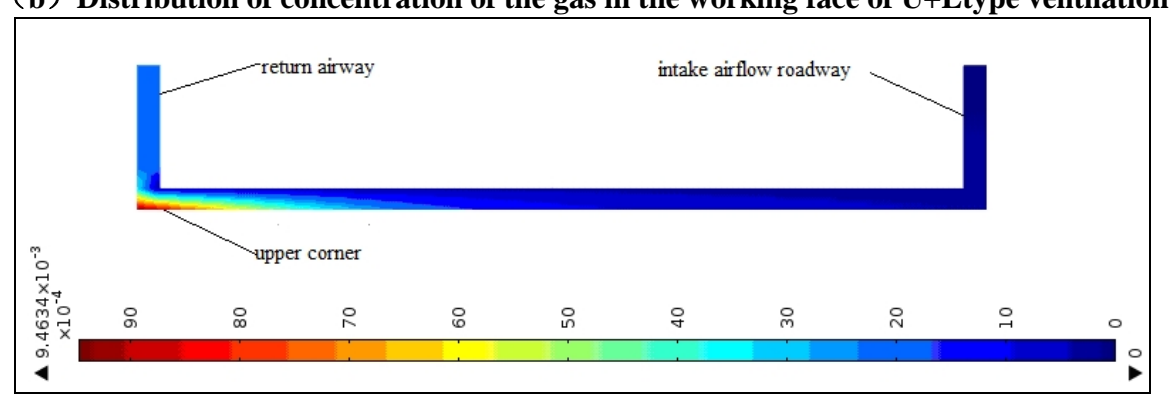

(c) Distribution of concentration of the gas in the working face of $U$ type + high drainage roadway

Fig. 4 gas concentration distribution in working face

The working face gas concentration distribution map shows that working face gas accumulation may occur mainly in the area near the corner, which is the area that the coal mine needs to pay attention to, working face upper corner gas accumulation in two main reasons: Return air roadway and working face near the junction of the mining empty area gas and airflow in the outflow region, high gas concentration with airflow flowing from here, so that in the upper corner of the local gas agglomeration; in addition, the upper corner may be in the vortex state, which leads to a high concentration of gas to enter the air difficultly and can not be diluted, causing the top corner gas concentration overrun easily.

Under the condition of $U$ type ventilation, gas concentration in the upper corner of working face reached $6.62 \%$, according to the provisions of "coal mine safety regulations", the gas concentration of tailentry may not be higher than $1 \%$, so we need other gas control measures. Under the condition of $U$ 
type ventilation plus tail gas special drainage roadway, gas tail roadway emission high concentration gas, at the same time, tail roadway of airflow guiding role of the working surface of fresh wind left toward the goaf side of the flow, the return air side of gas concentration is decreased greatly, on the working surface of the corner gas concentration decreased to $0.81 \%$. Under the condition of U-type ventilation plus to high drainage roadway ventilation, high drainage roadway drainage gas with high concentration, at the same time, drainage to the guiding role of the negative pressure of air make a lot of pressure relief gas influx into the return air lane side of the cracked area after entering high drainage roadway, the return air side gas horizon gather raised to a high level suction roadway drainage opening position, the upper corner gas concentration decreased to $0.94 \%$.

\section{Conclusions}

According to the goaf overburden caving broken characteristics, puts forward the goaf mining fissure rectangular terrace geometric model;and then the working face and goaf gas migration coupling model are put forward, the Navier Stokes flow and mining empty area of the Brinkman flow organic link in uniform flow field, and the introduction of the solute diffusion equation of translation the whole working face and goaf flow field distribution and the gas concentration distribution, more in line with the actual.Using COMSOL Multiphysics for nonlinear coupling calculation of three kinds of ventilation model, the results show that high gas special drainage tunnel on goaf gas accumulation effect is obvious, the risk can decrease goaf gas influx of working face caused by gas, but also provide the basis for high gas tunnel arrangement.

Due to the complexity of the problem, want to completely in accordance with the actual situation of goaf gas concentration distribution were simulated is impossible, specific to this model, gob permeability, porosity, and gas emission boundary can set up to be more close to the real, and need to continue to carry out in-depth research work.

\section{Reference:}

[1] Zhou Shining, Lin Baiquan. Coal Seam Gas Occurrence flow theory [M]. Beijing: China Coal Industry Publishing House, 1998.

[2] Hu Qianting. CMM and Gas Disaster Prevention [M]. Xuzhou: China University of Mining and Technology Press, 2007.

[3] money Domingo, Miao Hip Hing. Critical layer theory [M] strata control. Xuzhou: China University of Mining and Technology Press, 1998.

[4] Yuan Liang. High Gas Mine complex geological conditions for safe and efficient exploration of key technologies [J]. COAL SOCIETY, 2006,31 (2): 174-178.

[5] Yuan Liang. Theory relief gas extraction and coal mining technology and gas system [J]. COAL SOCIETY, 2009,34 (1): 1-8.

[6] Lizong Xiang, Sun Guangyi, Wang Jibo value of mining goaf heterogeneous porous media flow pattern simulation [J] Rock Mechanics and Engineering, 2001,20 (S2):.. 1578-1581.

[7] Yang Tianhong, Owen wide, Zhu Wancheng, and other mine rock failure water inrush mechanism and nonlinear percolation model of [J] Rock Mechanics and Engineering, 2008,27 (7): 1411-1416.

[8] Yang Tianhong, Owen wide, Zhu Wancheng, and other coal seam gas drainage relief dynamic process of gas - solid coupling model [J]. Rock and Soil Mechanics, 2010,31 (7): 2247-2252.

[9] Hu Qianting, Liangyun Pei, Liu see the goaf gas flow patterns CFD simulations [J] coal, 2007,32 (7): 719-723. 